Supplementary for

\title{
A Mechanically Reprogrammable Pancharatnam-Berry Metasurface for Microwaves
}

Supplementary 1. Details of the stepping motor

Supplementary 2. Details of the metasurface template

Supplementary 3. Simulation results

Supplementary 4. Measured phase responses at different frequencies

Supplementary 5. Compensation phase

Supplementary 6. Metalensing at different focus lengths

Supplementary 7. OAM component of the measured vortex beams

Supplementary 8 . Noise analysis of focused vortex beam generations

Supplementary 9. Modified Gerchberg-Saxton algorithm

Supplementary 10. Holographic imaging for LCP incidence

Supplementary 11. Polarization-insensitive Metalensing

Supplementary 12. Polarization/frequency-multiplexed metalensing

Supplementary 13. Multidimensional manipulation by mechanical approaches

Supplementary 14. Comparison of different levels of phase control

Supplementary 15. Experimental setup 


\section{Supplementary 1. Details of the stepping motor}

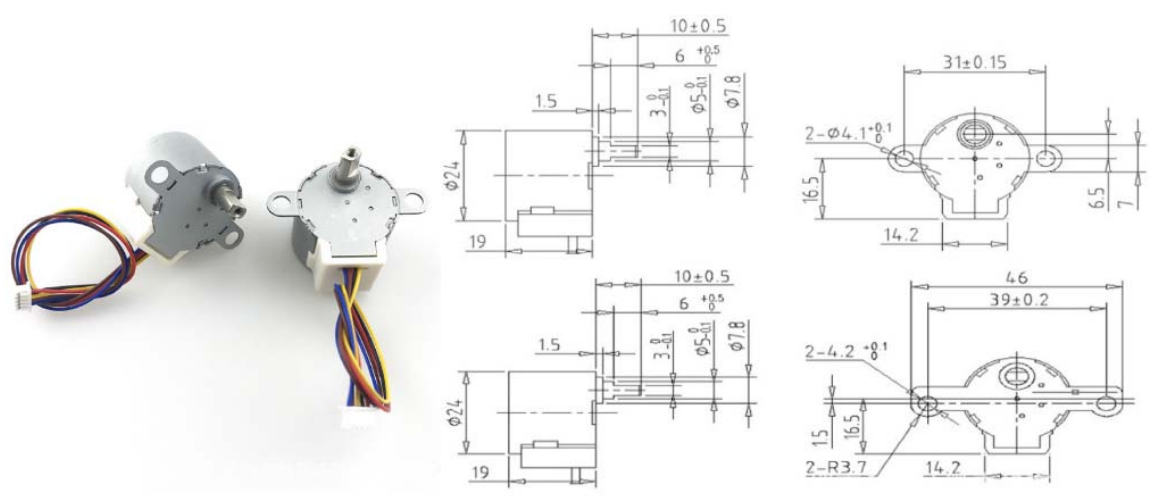

Figure S1. Photo and detailed geometric parameters $(\mathrm{mm})$ of the adopted stepping motor.

\begin{tabular}{l|l}
\hline Rated voltage & Motor terminal voltage of $5.0 \mathrm{~V} \mathrm{DC}$ \\
\hline Number of Phase & 4 phases \\
\hline Drive mode & $1-2$ phase excitation unipolar drive \\
\hline Step angle & $5.625^{\circ} / 64$ (out shaft), gear reduction ration $1 / 64$ \\
\hline Resistance per phase & $40 \Omega \pm 15 \% /$ phase $@ 25^{\circ} \mathrm{C}$ \\
\hline Power dissipation & $<600 \mathrm{~mW}$ \\
\hline Max response frequency & $\geq 1000 \mathrm{pps}$ \\
\hline Max starting frequency & $\geq 500 \mathrm{pps}$ \\
\hline Pull in torque & $\geq 20 \mathrm{mN} . \mathrm{m} / 5 \mathrm{VDC} 550 \mathrm{~Hz}$ \\
\hline Weight & $28 \mathrm{~g}$ \\
\hline Endurance test & The motor meets the requirements after 3000 hours and \\
$90^{\circ}$ rotation in the positive and negative directions under \\
the load of $5 \mathrm{VDC}$ and $100 \mathrm{gf}$ cm
\end{tabular}

Table S1. Electrical performance. These stepping motors were brought from a commercial company named Shenzhen Wei-Chen-Hao Motor Limited Company in Shenzhen, China. 


\section{Supplementary 2. Details of the reprogrammable metasurface}

a

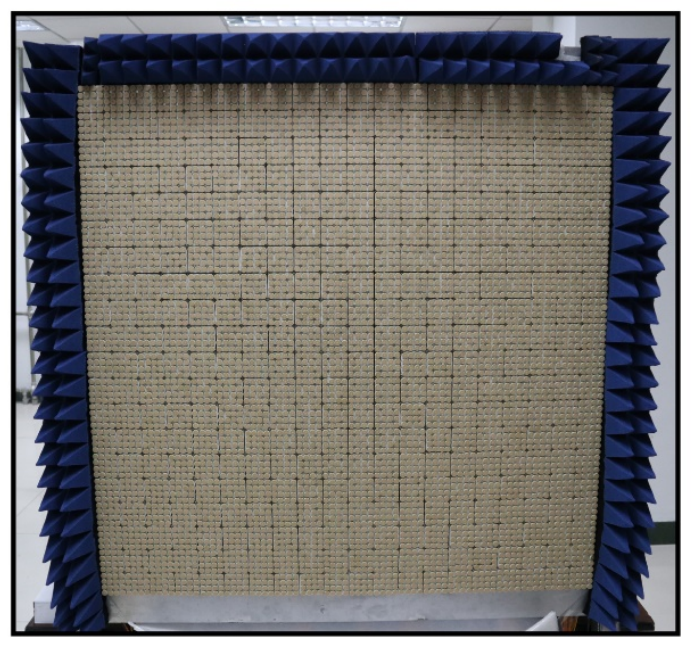

C

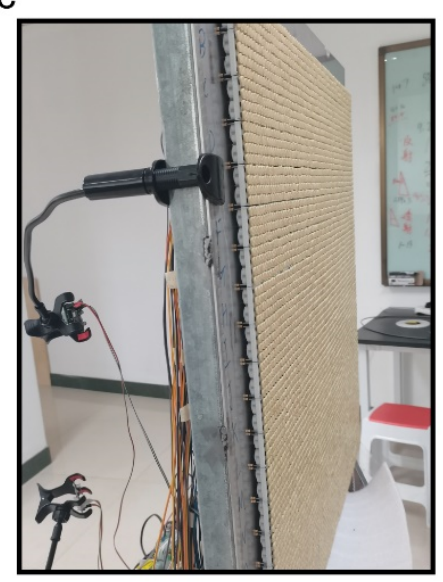

b
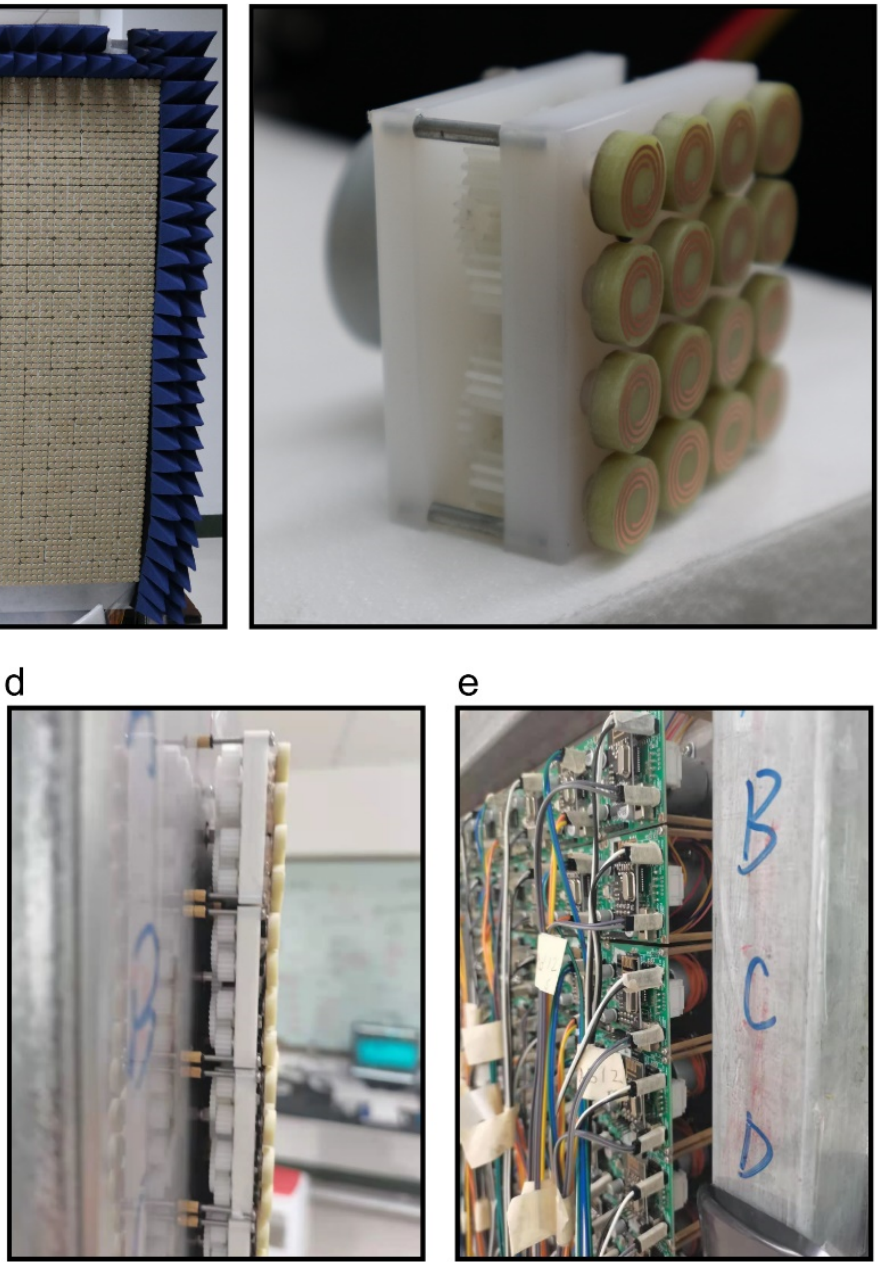

e

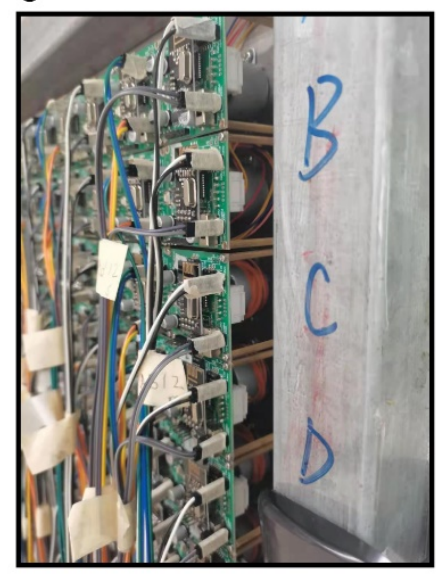

Figure S2. (a) Front view of the metasurface template. (b) Photo of a single super-cell, gear set and meta-atoms attached in the back and front sides, respectively, of a plastic board with size of $42.5 \times 42.5 \mathrm{~mm}^{2}$. (c-e) In the experiment, all super-cells are attached on a steel board, the gear set and meta-atoms are located on the front side, while the addressed circuits and stepping motors are located on the back side. In the circuit, a wireless receiver (NRF24L01) is adopted to receive a control signal from the host computer, and a single chip microcontroller (STC8A8K64S4A12) is adopted to input pulse signals to the stepping motor.

The meta-atoms were fabricated by a commercial company named Shenzhen HuaBang-Xin Circuit Technology in Shenzhen, China, following the traditional Printed Circuit Board (PCB) fabrication technology. The technological details include: FR4 
material, board thickness $3.0 \mathrm{~mm}$, copper weight 1oz, no solder mask.

The gears were brought from online shop Ku-Wan-Ke-Chuang at www.taobao.com. These gears are standard toy gears made from plastic.

The addressed circuits were fabricated by a commercial company named Shenzhen Fan-Yi Circuit Technology http://www.fany-eda.com/ in Shenzhen, China, following the traditional PCB fabrication technology. 


\section{Supplementary 3. Simulation results}

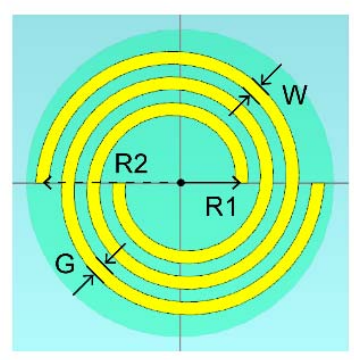

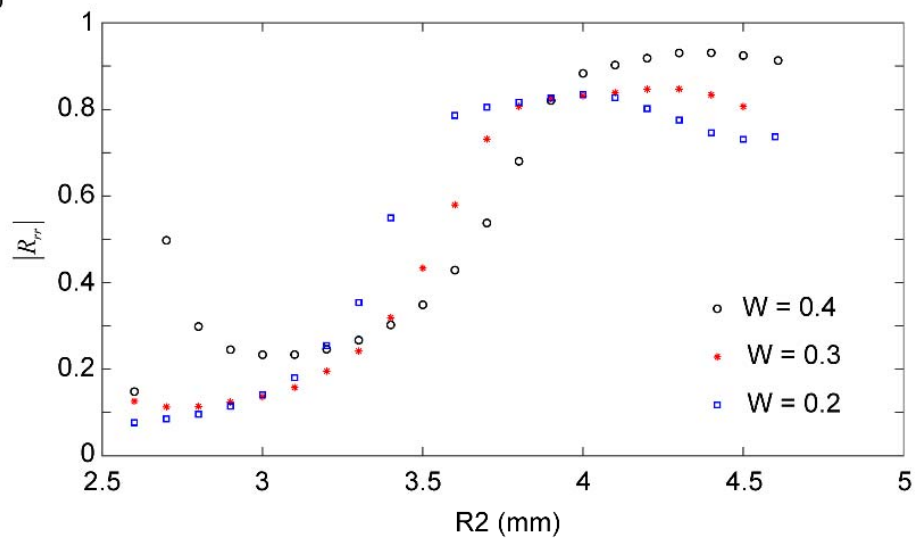

c

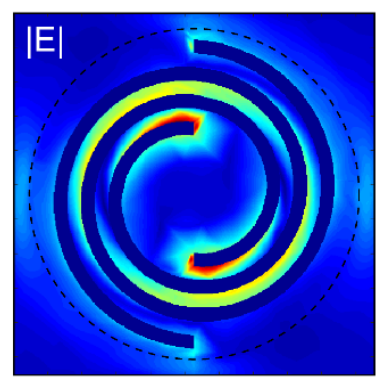

d

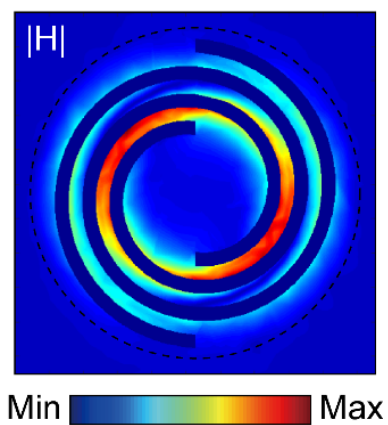

Figure S3. Simulated results using the frequency-domain solver of commercial software CST Micro-wave Studio. (a) The inner radius (R1) and the outer radius (R2) are defined as the distances from the meta-atom center to the centers of the innermost and outmost metal strip, respectively. The gap $(G)$ between adjacent metal strips and the width (W) of the metal strip are set as the same. In this condition, $\mathrm{R} 2=\mathrm{R} 1+\mathrm{W}^{*} 6$. (b) Simulated results of $\left|R_{r r}\right|$ in the cases of $\mathrm{W}=0.2 \mathrm{~mm}, 0.3 \mathrm{~mm}$, and $0.4 \mathrm{~mm}$, scanning R2 from $2.6 \mathrm{~mm}$ to $4.6 \mathrm{~mm}$. (c, d) Simulated electric and magnetic field distribuitons. In simulation, the boundaries are set as unit cell and RCP waves impinge onto the metaatom from the Archimedean spirals side. The field distributions are obtained by setting field monitors of $7 \mathrm{GHz}$ at the surface of meta-atom. 


\section{Supplementary 4. Measured phase responses at different frequencies}

a

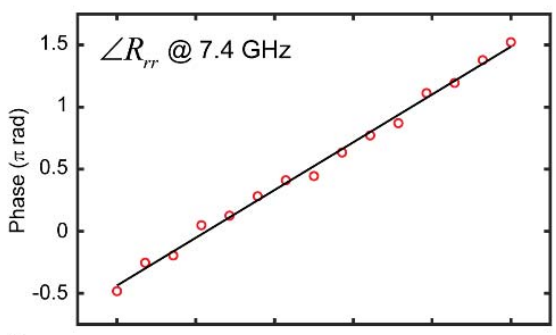

C

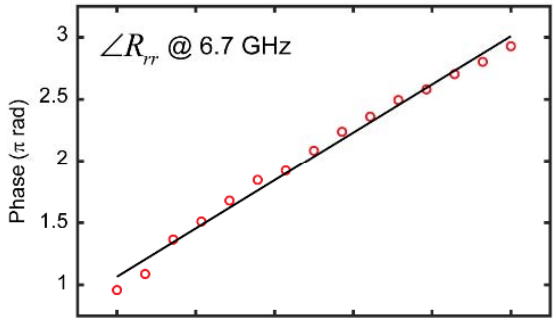

e

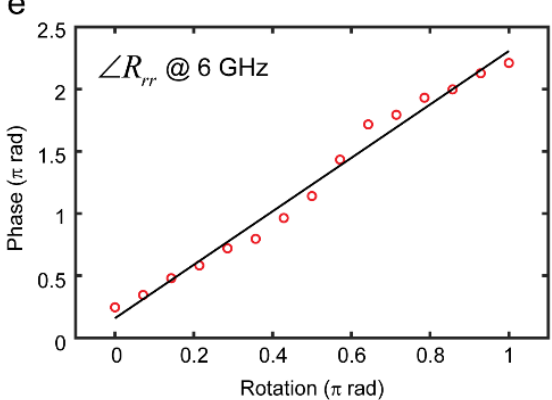

b

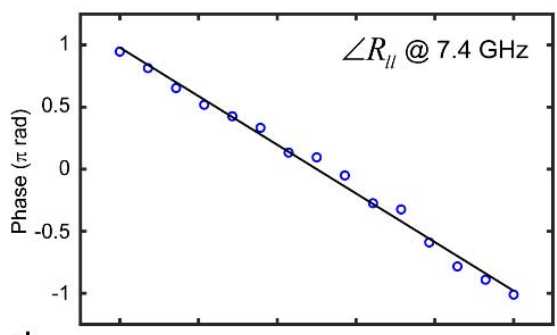

d

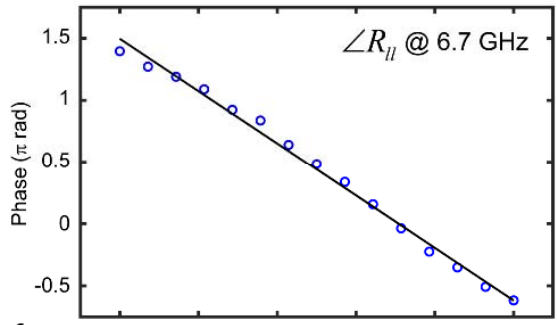

f

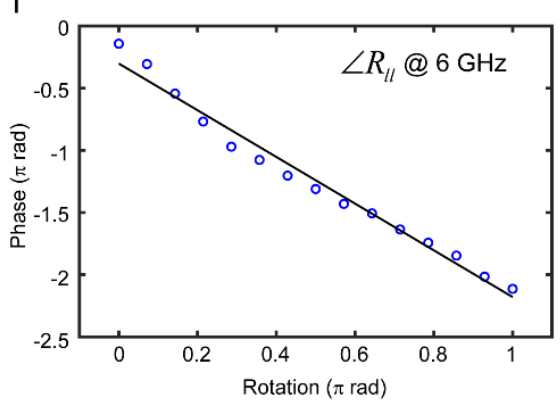

Figure S4. Extracted phase responses of $R_{r r}$ and $R_{l l}$ from the measurements, respectively, at $7.4 \mathrm{GHz}, 6.7 \mathrm{GHz}$, and $6 \mathrm{GHz}$. (a, c, e) The fitting gradients of $R_{r r}$ are $1.924,1.9438$, and 2.1482, respectively, for the experimental results at $7.4 \mathrm{GHz}, 6.7$ GHz, and $6 \mathrm{GHz}$. (b, d, f) The fitting gradients of $R_{l l}$ are $-1.9603,-2.1146$, and -1.8775 , respectively, for the experimental results at $7.4 \mathrm{GHz}, 6.7 \mathrm{GHz}$, and $6 \mathrm{GHz}$. 


\section{Supplementary 5. Compensation phase}

a

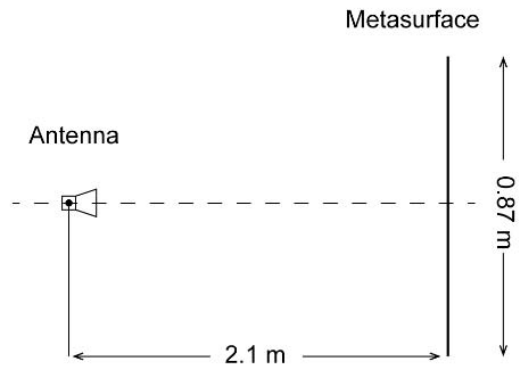

b

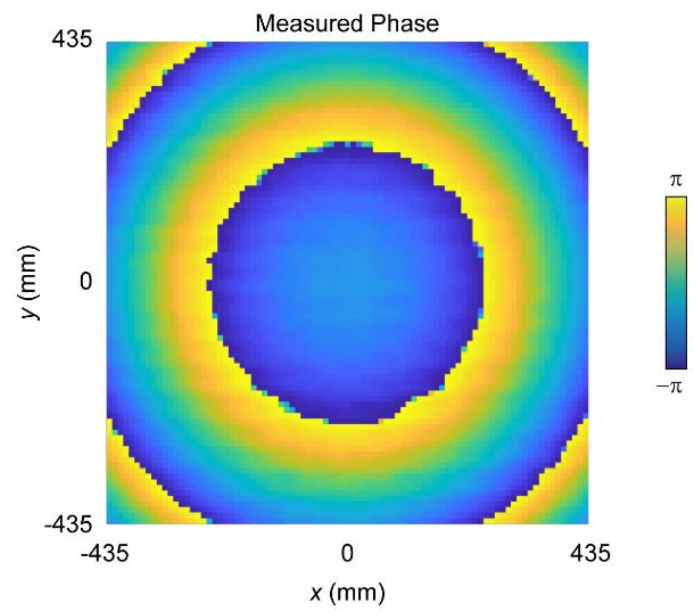

Figure S5. (a) In our experiment, the metasurface size is $870 \times 870 \mathrm{~mm}^{2}$. The feeding antenna is mounted at a distance of $2.1 \mathrm{~m}$ away from the metasurface, which is not in the Fraunhofer zone $R=2 L^{2} / \lambda$, here $L$ is the maximum electrical length of the antenna and $\lambda$ is the working wavelength. In this configuration, the feeding antenna cannot provide a quasi-planar wavefront incident on the metasurface. (b) Electric field distribution at $7 \mathrm{GHz}$ at the metasurface plane. The phase difference between the center and edges of the measurface is larger than $2 \pi$, which induce distortion of the target functionality. Therefore, in the experiments all the designs are combined with a compensation phase by treating the antenna as a point source. 


\section{Supplementary 6. Metalensing at different focal lengths}
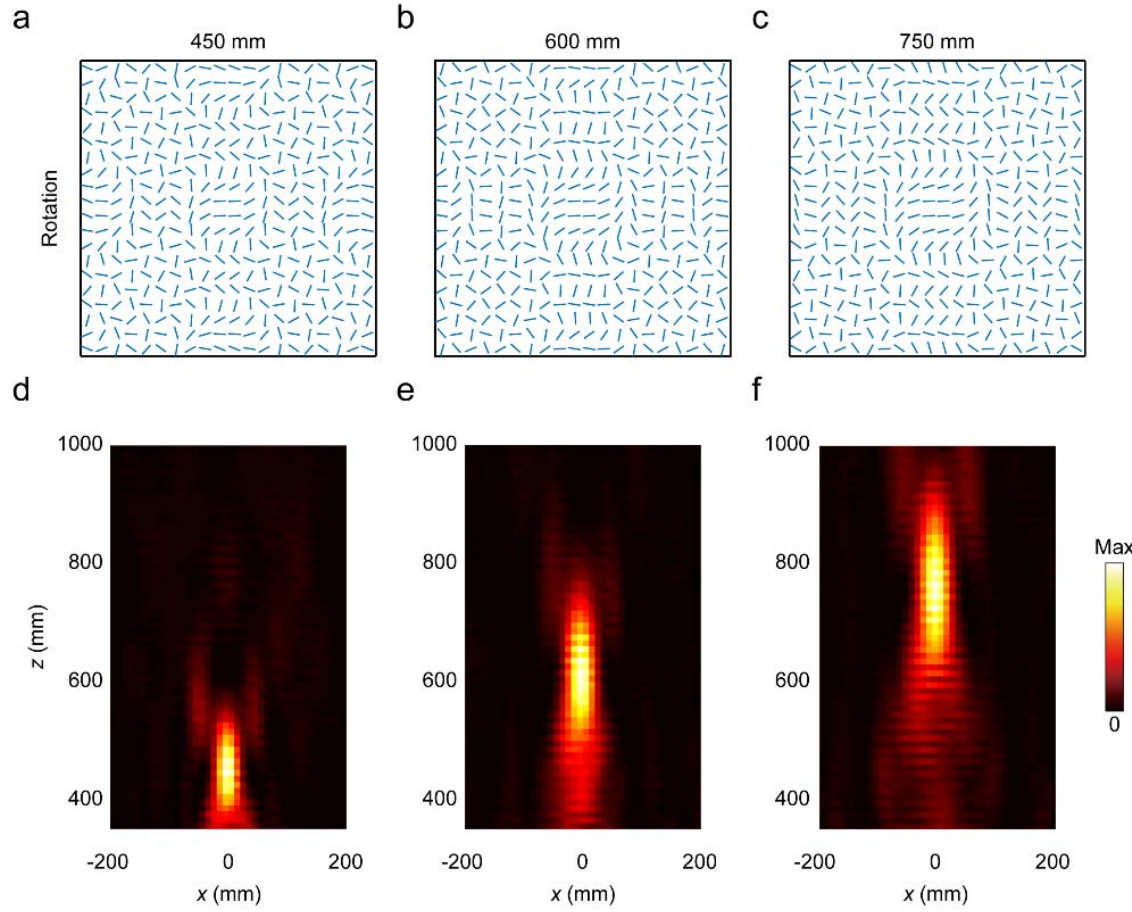

Figure S6. The desired phase distribution $\Phi(x, y)$ to realize metalensing at $f_{0}\left(x_{0}, y_{0}, z_{0}\right)$ can be calculated as $\Phi(x, y)=\frac{2 \pi}{\lambda}\left(\sqrt{\left(x-x_{0}\right)^{2}+\left(y-y_{0}\right)^{2}+z_{0}^{2}}-z_{0}\right)$, here $\lambda$ is the target operating wavelength. Since our proposed metasurface can directly control the phase distribution, metalensing at different focal lengths can be realized. (a-c) Required orientation profiles to realize metalens with target focus lengths are $(0,0,450 \mathrm{~mm}),(0$, $0,600)$, and $(0,0,750 \mathrm{~mm})$, respectively. Here the operation wave is RCP at $7 \mathrm{GHz}$. (b, $\mathrm{e}, \mathrm{h})$ Measured electric field intensities $\left(\left|R_{r r}\right|^{2}\right)$ at $7 \mathrm{GHz}$ on the $x z$-plane at $y=0 \mathrm{~mm}$. 


\section{Supplementary 7. OAM component of the measured vortex beams}

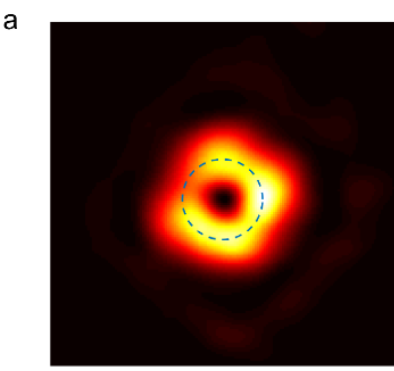

d

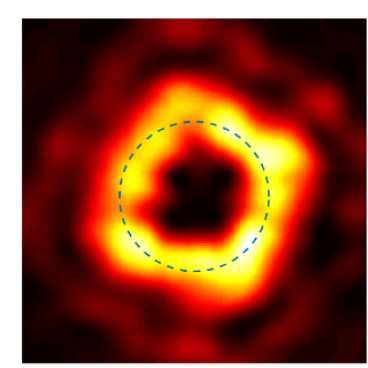

b

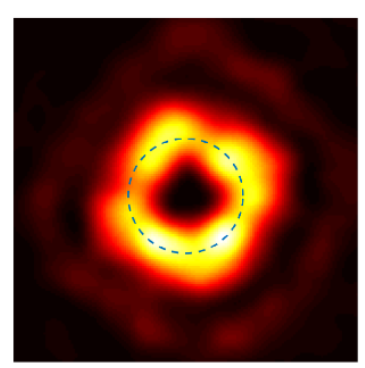

d

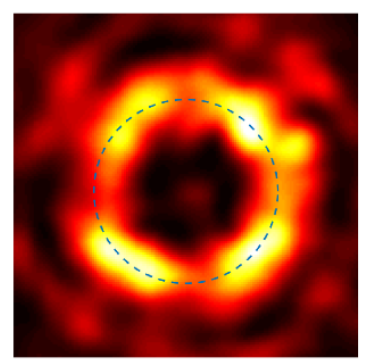

Figure S7. We extract the complex field distributions $E_{i}$ along the circles as marked by dashed lines, respectively, here $i=\mathrm{a}, \mathrm{b}, \mathrm{c}, \mathrm{d}$ represent the results extracted from (a-d). As different OAM components (with different topological charges) are perpendicular to each other and thus can be seen as a group of orthogonal basis, we can expand the extracted field using this basis to obtain the strength of each OAM component $\left|S_{n}\right|$. Namely, roughly get the amplitude of each OAM component by doing the following integration:

$$
\left|S_{n}\right|=\left|\int_{0}^{2 \pi} E_{i} e^{i n \varphi} \mathrm{d} \varphi\right|
$$

Here $n$ is the topological charge of target OAM component and $\varphi$ is the azimuthal angle. 


\section{Supplementary 8. Noise analysis of focused vortex beam generations}

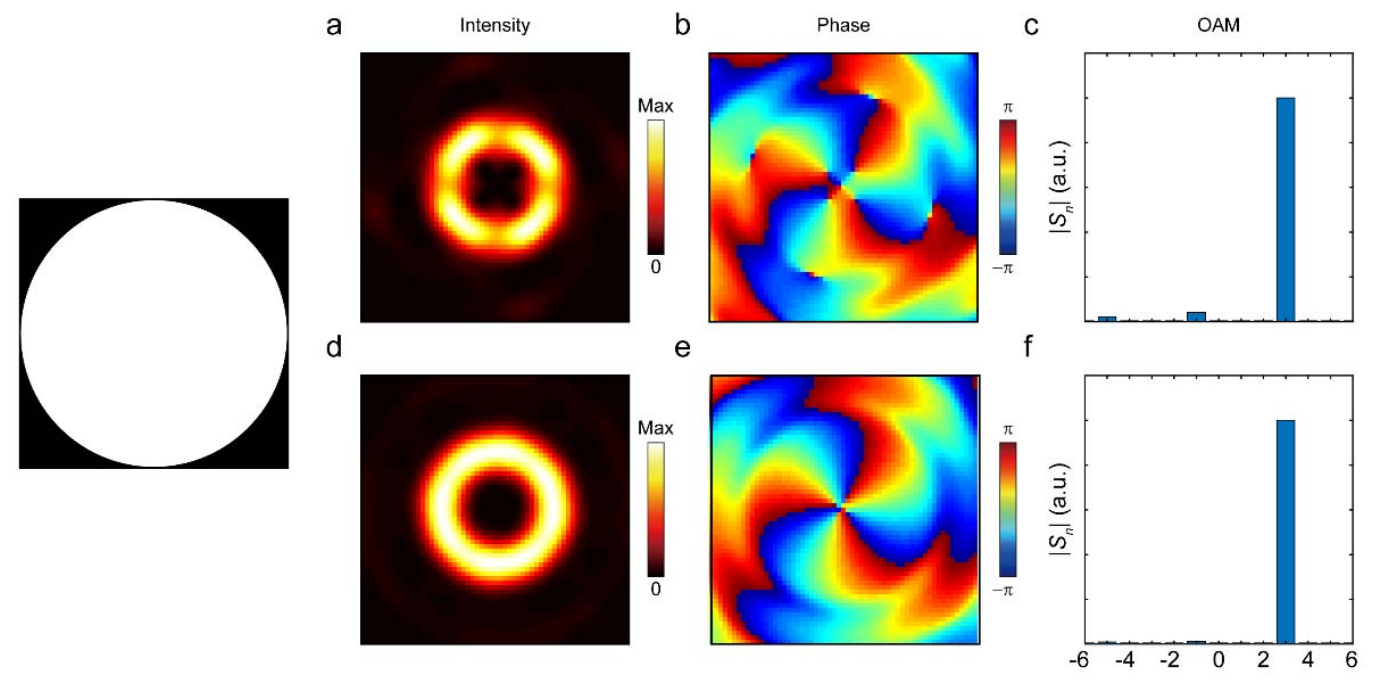

Figure S8. (a-c) The calculated results of OAM beam with topological charge of 3 , which are calculated from the square-shaped metasurface that same as used in the manuscript. In comparison, if we set the metasurface amplitude response out of the inscribed circle as 0 , as depicted by the back area in the left panel, the corresponding calculated results (d-f) exhibit much purer OAM beam. The experimental errors also lead to the interference with unwanted OAM beams. Limited by our current experiment condition, the results shown in the manuscript were not obtained in the microwave anechoic chamber. Despite we placed as much microwave absorbers as possible around the experiment setup during the measurement, there still existed some unavoidable scatterings. These measurement noises along with the fabrication and assembling induced metasurface variations directly leaded to the experimental errors. 


\section{Supplementary 9. Modified Gerchberg-Saxton algorithm}

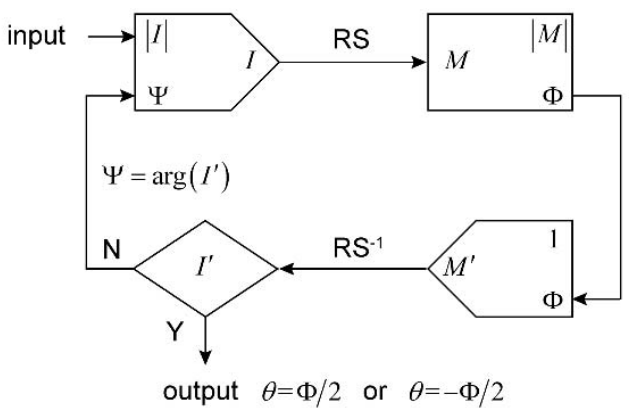

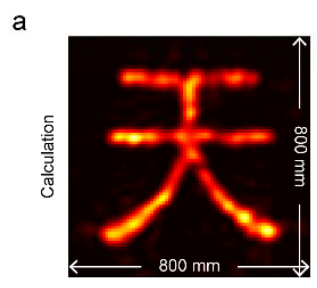

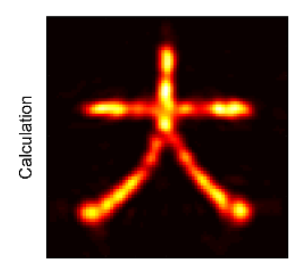

b

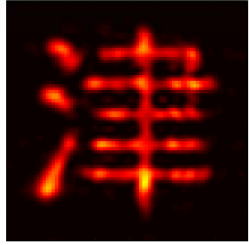

$f$

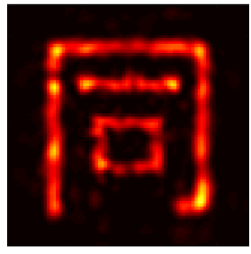

C

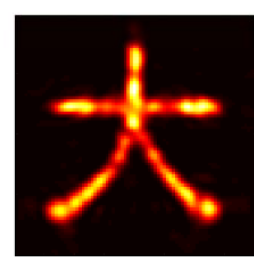

9

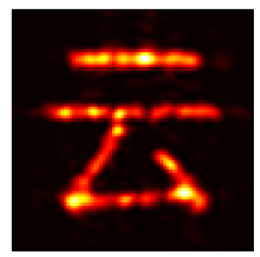

d
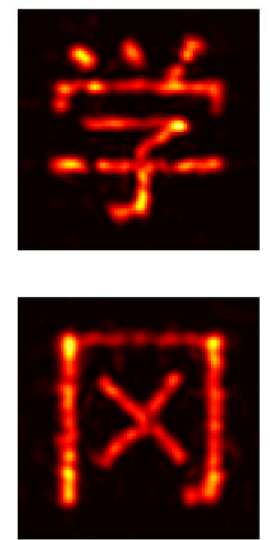

Figure S9. Since our metasurface is $870 \mathrm{~mm}$ wide (along both the $x$ and $y$ directions) and use an imaging distance of $z=600 \mathrm{~mm}$, it does not satisfy the Fresnel approximation in diffraction optics. Thus, the holographic image generation algorithm used in the manuscript is a modified Gerchberg-Saxton algorithm, where the conventionally used Fresnel diffraction formula is replaced by the RayleighSommerfeld diffraction formula (Refs. 62 and 63 in manuscript). Suppose that the metasuface and target image are located at two $x y$-planes with a relative distance $\Delta z=$ $600 \mathrm{~mm}$ along the $z$-axis. In the flow chart, the input $|I|$ is the virtual amplitude distribution of target image, in conjunction with a virtual phase distribution $\Psi$ ( $\Psi$ is set as a random phase distribution in the first loop), the complex amplitude distribution of target image can be calculated as $I=|I| \exp (i \Psi)$. The diffraction of the virtual image at a metasurface supercell located at $\left(x_{m}, y_{m}\right)$ can be calculated as:

$$
M\left(x_{m}, y_{m}\right)=\frac{1}{i \lambda} \sum_{x_{i}} \sum_{y_{i}} I\left(x_{i}, y_{i}\right) \frac{\Delta z \exp \left(i k r_{i}\right)}{r_{i}}
$$


here $\left(x_{i}, y_{i}\right)$ is the position of virtual point source that comprise the target image, $k=$ $2 \pi / \lambda$ is the wave number, and $r_{i}=\sqrt{\left(x_{i}-x_{m}\right)^{2}+\left(y_{i}-y_{m}\right)^{2}+(\Delta z)^{2}}$ is the distance between points $\left(x_{m}, y_{m}\right)$ and $\left(x_{i}, y_{i}\right)$. By calculating all the supercells, one can get the complex amplitude distribution $M=|M| \exp (i \Phi)$ at the metasurface plane, and we define this operation as RS. Since our metasurface has the normal amplitude response, the $M$ is normalized as $M^{\prime}=\exp (i \Phi)$. Next, the holographic complex amplitude at a point $\left(x_{i}\right.$, $\left.y_{i}\right)$ in the imaging plane can be calculated as:

$$
I^{\prime}\left(x_{i}, y_{i}\right)=\frac{1}{i \lambda} \sum_{x_{m}} \sum_{y_{m}} M^{\prime}\left(x_{m}, y_{m}\right) \frac{\Delta z \exp \left(-i k r_{m}\right)}{r_{m}},
$$

here $r_{m}=\sqrt{\left(x_{m}-x_{i}\right)^{2}+\left(y_{m}-y_{i}\right)^{2}+(\Delta z)^{2}}$. By calculating all the points at image plane, one can get the complex amplitude distribution of holographic image $I^{\prime}=\left|I^{\prime}\right| \exp (i \Psi)$, and we define this operation as $\mathrm{RS}^{-1}$. Then comparing the holographic image $\left|I^{\prime}\right|$ with target image $|I|$ by:

$$
Q=\sum_{x_{i}} \sum_{y_{i}}\left|\left(\left|I^{\prime}\left(x_{i}, y_{i}\right)\right|^{2}-\left|I\left(x_{i}, y_{i}\right)\right|^{2}\right)\right| \text {. }
$$

If the holographic image meets the requirement of $Q$ is small enough, the iteration terminates and output the required rotation angle profile $\theta=\Phi / 2$ or $\theta=-\Phi / 2$ depending on the target work polarization is RCP or LCP. If not, by combining the desired amplitude distribution $|I|$ with the calculated phase distribution $\Psi$, the circulation proceeds. Since the $Q$ gradually converges as the iteration process, the different designs shown in Fig. 5 were all obtained by performing 200-loops, rather than setting a certain comparing value for $Q$. (a-h) Calculated holographic images $\left|I^{\prime}\right|^{2}$ of “天津大学” and “大同云冈”. In calculation, we treat each meta-atom as point source with ideally designated hologram phase profiles, and the calculated holographic images are in good agreements with target Chinese sentences. 


\section{Supplementary 10. Holographic imaging for LCP incidence}
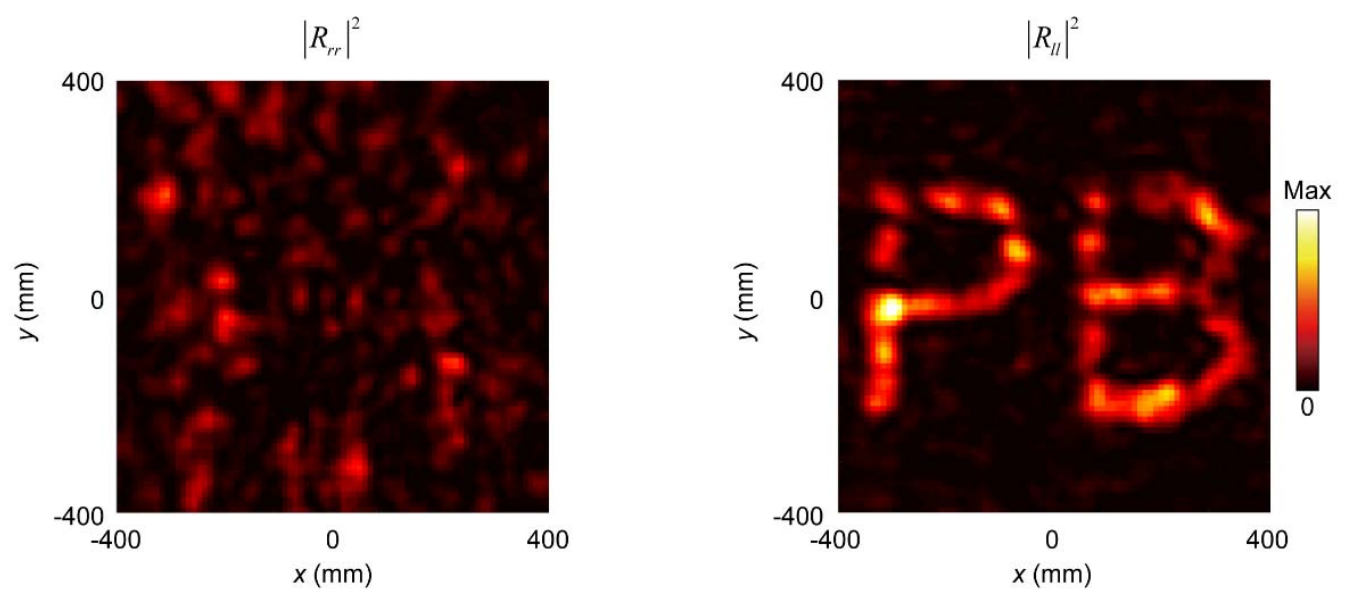

Figure S10. Based on the modified Gerchberg-Saxton algorithm, holographic imaging of words "PB" is designed to work under the LCP incidence. The figure shows corresponding experiential results, i.e., measured electric field intensities $\left|R_{r r}\right|^{2}$ and $\left|R_{l l}\right|^{2}$ at $7 \mathrm{GHz}$, respectively, in the $x y$-plane of $z=600 \mathrm{~mm}$. 


\section{Supplementary 11. Polarization-insensitive Metalensing}

a

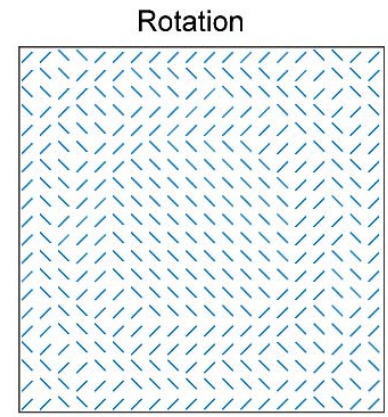

b

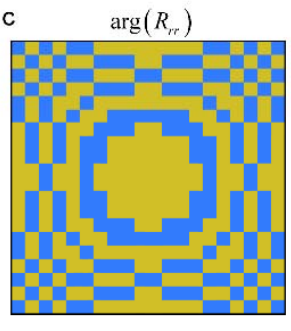
g
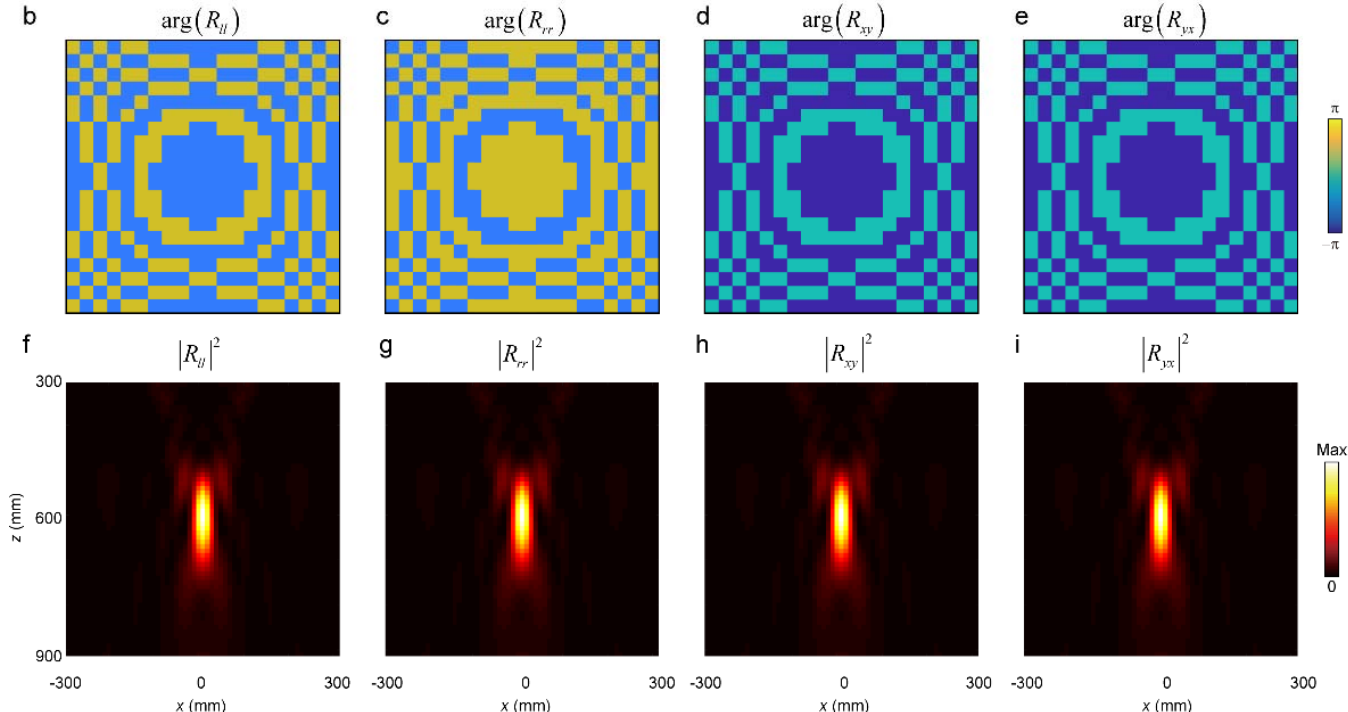

Figure S11. (a) The rotation profile of meta-atoms to achieve metalens at $(0,0,600$ $\mathrm{mm}$ ), where the orientation angles are binarily arranged with $-\pi / 4$ and $\pi / 4$. In this configuration, the corresponding phase distributions of different reflected crosspolarizations are illustrated in (b-e), where the complex amplitudes satisfy $R_{l l}=e^{i \pi} R_{l l}=$ $i R_{x y}=i R_{y x}$. In other words, with a relative phase difference, they can all meet the phase requirement of metalensing at $(0,0,600 \mathrm{~mm})$. The corresponding calculated intensity distributions of reflected cross-polarized fields shown in (f-i) can focus at the same spot. 


\section{Supplementary 12. Polarization/frequency-multiplexed metalensing}

a
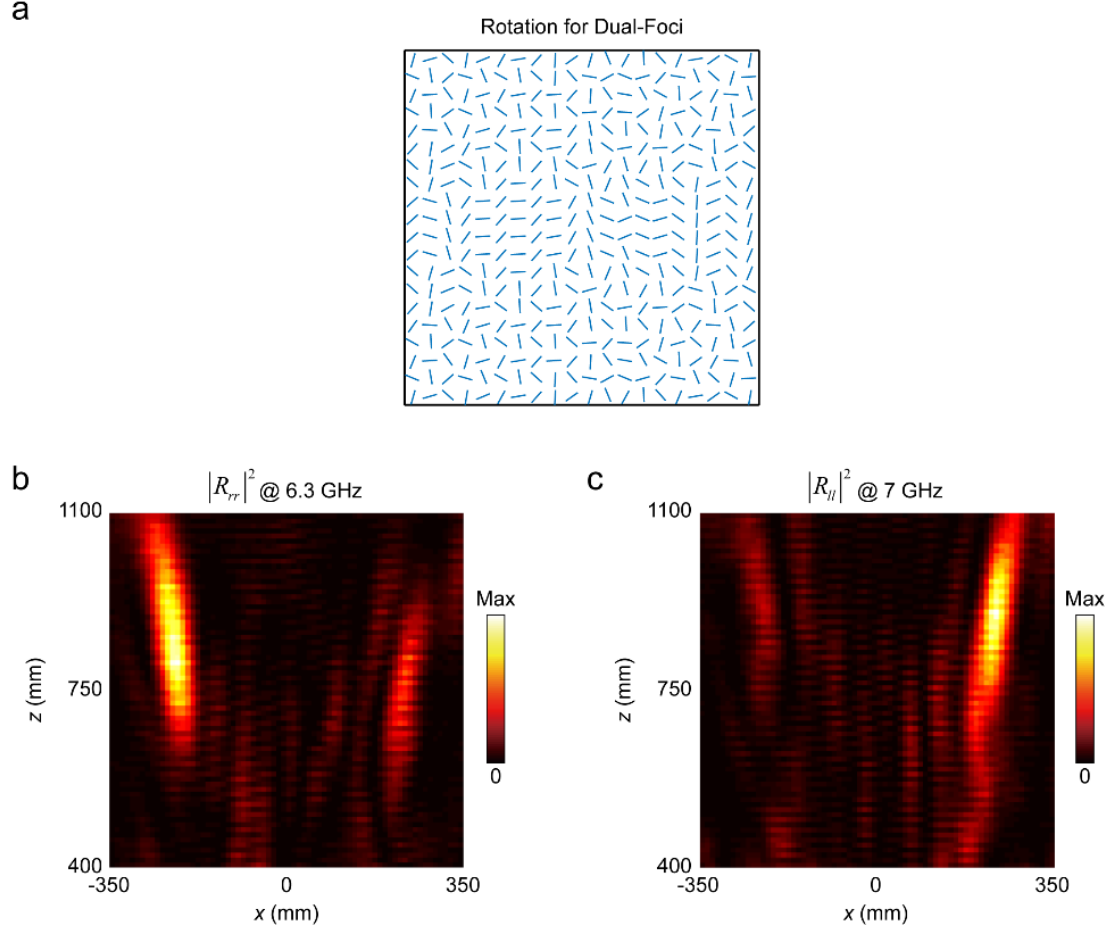

Figure S12. (a) Required rotation profile for focusing $R_{r r}$ of $6.3 \mathrm{GHz}$ at $(-250,0,850$ $\mathrm{mm}$ ) and focusing $R_{l l}$ of $7 \mathrm{GHz}$ at $(250,0,900 \mathrm{~mm}) .(\mathrm{b}, \mathrm{c})$ The measured intensity distributions of $\left|R_{r r}\right|^{2}$ and $\left|R_{l l}\right|^{2}$, respectively, at $6.3 \mathrm{GHz}$ and $7 \mathrm{GHz}$. 


\section{Supplementary 13. Multidimensional manipulation by mechanical approaches}

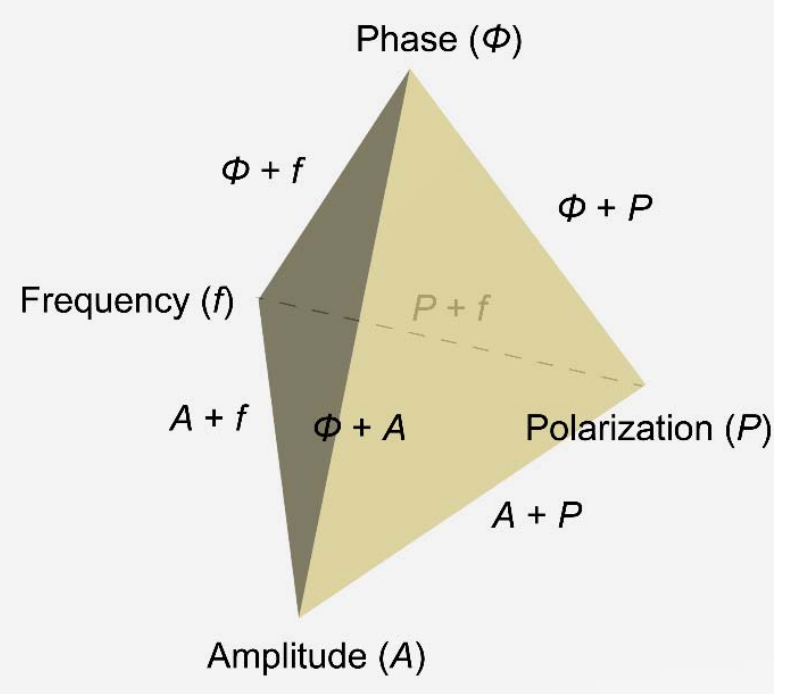

Figure S13. Phase, amplitude, polarization and frequency are the four fundamental characteristics of electromagnetic waves. The recent paper (Ref. 24 in main text) reviews the artificial microstructure-based multidimensional manipulations of electromagnetic fields in details. Following similar steps, here we discuss the possibility of different kinds of multi-dimensional manipulations by our mechanical metasurface, and the possible solutions offered by the mechanical approach.
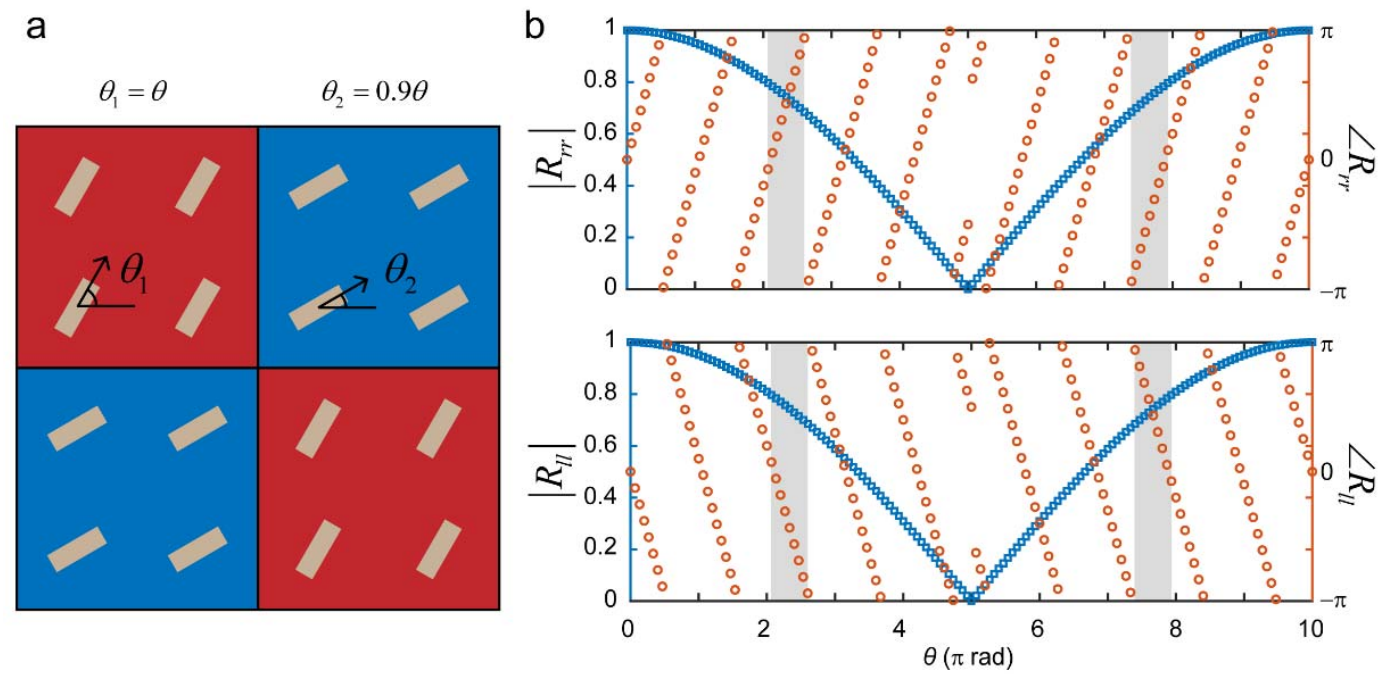

Figure S14. Simultaneous manipulation of phase and amplitude. A possible solution is to change the gear setting to assign different orientation angles (with a fixed ratio) to the PB meta-atoms in different diagonal blocks within a single super cell, as depicted by the red and blue squares in Fig. S14a. This will provide enough degrees of freedom 
to tune both the amplitude and phase of the scattering from the supercell. For example, let the orientation angles in red and blue squares be $\theta_{1}=\theta$ and $\theta_{2}=0.9 \theta$, respectively, with $\theta$ representing the rotation angle of step motor. The complex amplitudes can be expressed as:

$$
\begin{aligned}
& R_{r r}=C_{r r} \exp (1.9 i \theta) \cos (0.1 \theta), \\
& R_{l l}=C_{l l} \exp (-1.9 i \theta) \cos (0.1 \theta) .
\end{aligned}
$$

Here $C_{r r}$ and $C_{l l}$ are complex coefficients of $R_{r r}$ and $R_{l l}$, respectively. Specially, $C_{r r}=C_{l l}$ for the PB meta-atoms with in-plane mirror symmetry. Figure S14b illustrates the calculated amplitudes and phases, respectively, of $R_{r r}$ and $R_{l l}$. It is seen that simultaneous manipulation of phase and amplitude can be achieved. For instance, the amplitude range of $0.7-0.8$, as marked by the shadowed region, can cover the phase in the full $2 \pi$ range.

Simultaneous manipulation of phase and amplitude. In order to achieve simultaneous control of phase and polarization, we can alter the position of each supercell along the propagation axis by a distance of $s$ (Ref. 59 in main text). Then the phase of $R_{r r}$ and $R_{l l}$ can be calculated as $2 \theta+2 s k$ and $-2 \theta+2 s k$, respectively, with $k$ represents the wave number. Thus, independent phase control of different CPs can thus be achieved, which can provide independent control over phase and polarization state.

a

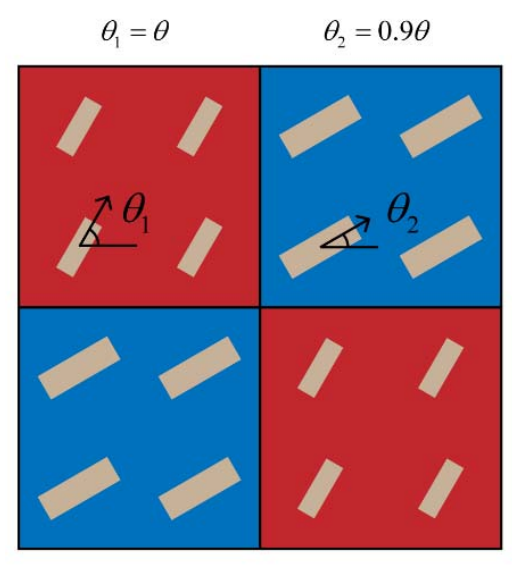

b

$$
C_{r r 1}=C_{l l 1}=i \quad C_{r 2}=C_{l l 2}=1
$$

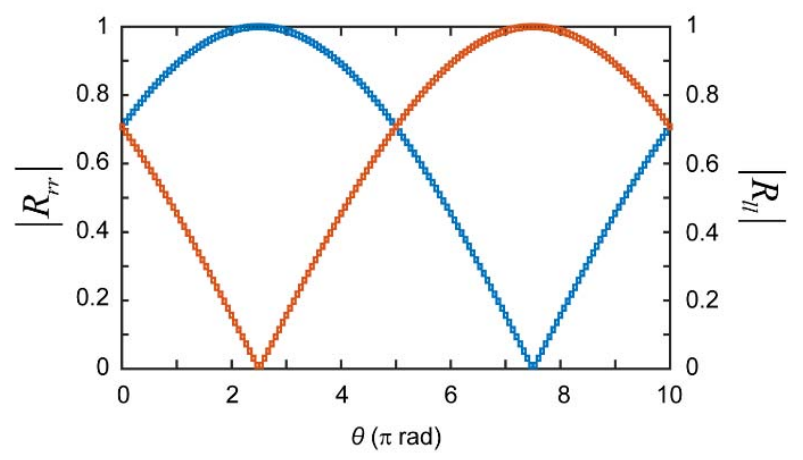

Figure S15. Simultaneous manipulation of polarization and amplitude. We can achieve this by exploiting the interference within a supercell, as shown in Fig. S15a. As 
example, let the orientation of the PB meta-atoms in red and blue squares be $\theta_{1}=\theta$ and $\theta_{2}=0.9 \theta$, respectively, with $\theta$ representing the rotation angle of step motor. Meanwhile, assuming that the PB meta-atoms in red and blue squares are different and the coefficients satisfy $C_{r r 1}=C_{l l 1}=i$ and $C_{r r 2}=C_{l l 2}=1$. Under this condition, the amplitudes for different CPs can be manipulated by the rotation angle $\theta$, as illustrated in Fig. S15b. Simultaneous manipulation of frequency and other dimensions are mainly achieved by nonlinear metasurfaces, which is kind of beyond the scope of mechanical tuning. 


\section{Supplementary 14. Comparison of different levels of phase control}

a

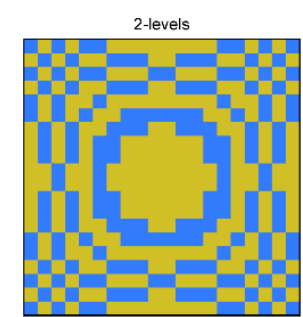

e
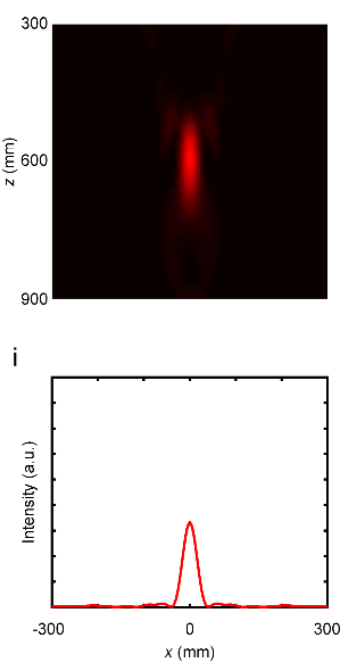

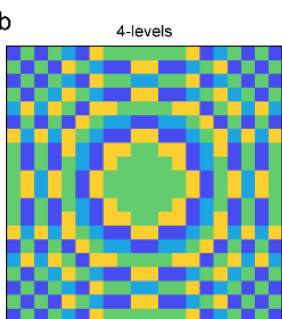

f
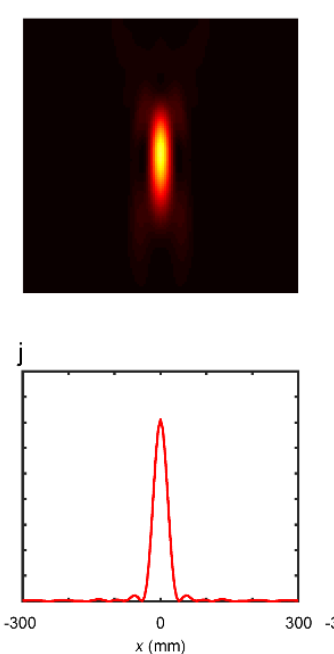
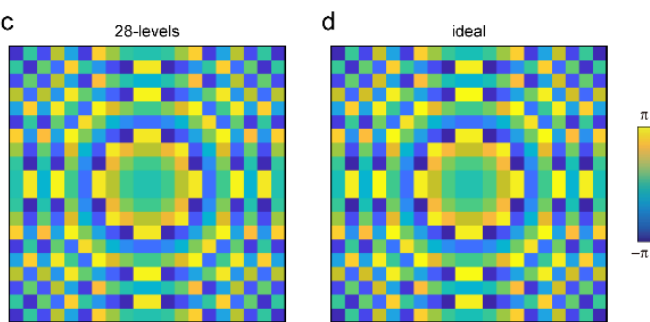

g
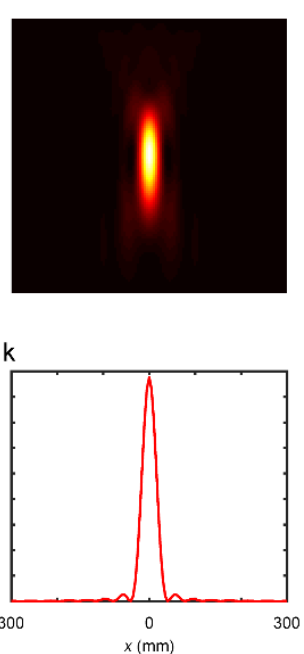

$\mathrm{h}$
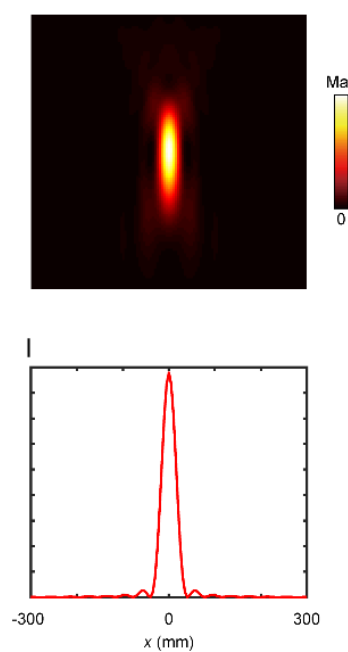

Figure S16. Numerical studies of different metalenses to quantitatively compare the performance of different phase control schemes. (a-d) Calculated phase distributions for the metalens with a focal at $(0,0,600 \mathrm{~mm})$, which is interpolated by $2,4,28$ (quasicontinuous), and ideal levels of phase control. (e-h) Calculated field intensities at the propagation plane (xz-plane). (i-j) Horizontal cuts of the focal spots at $z=600 \mathrm{~mm}$. Here the results indicate that the focusing intensity of quasi-continuous scheme is nearly the same as that of ideal one, and is about 2.61 and 1.25 times than that of 2 and 4 level phase control schemes, respectively. 


\section{Supplementary 15. Experimental setup}

a
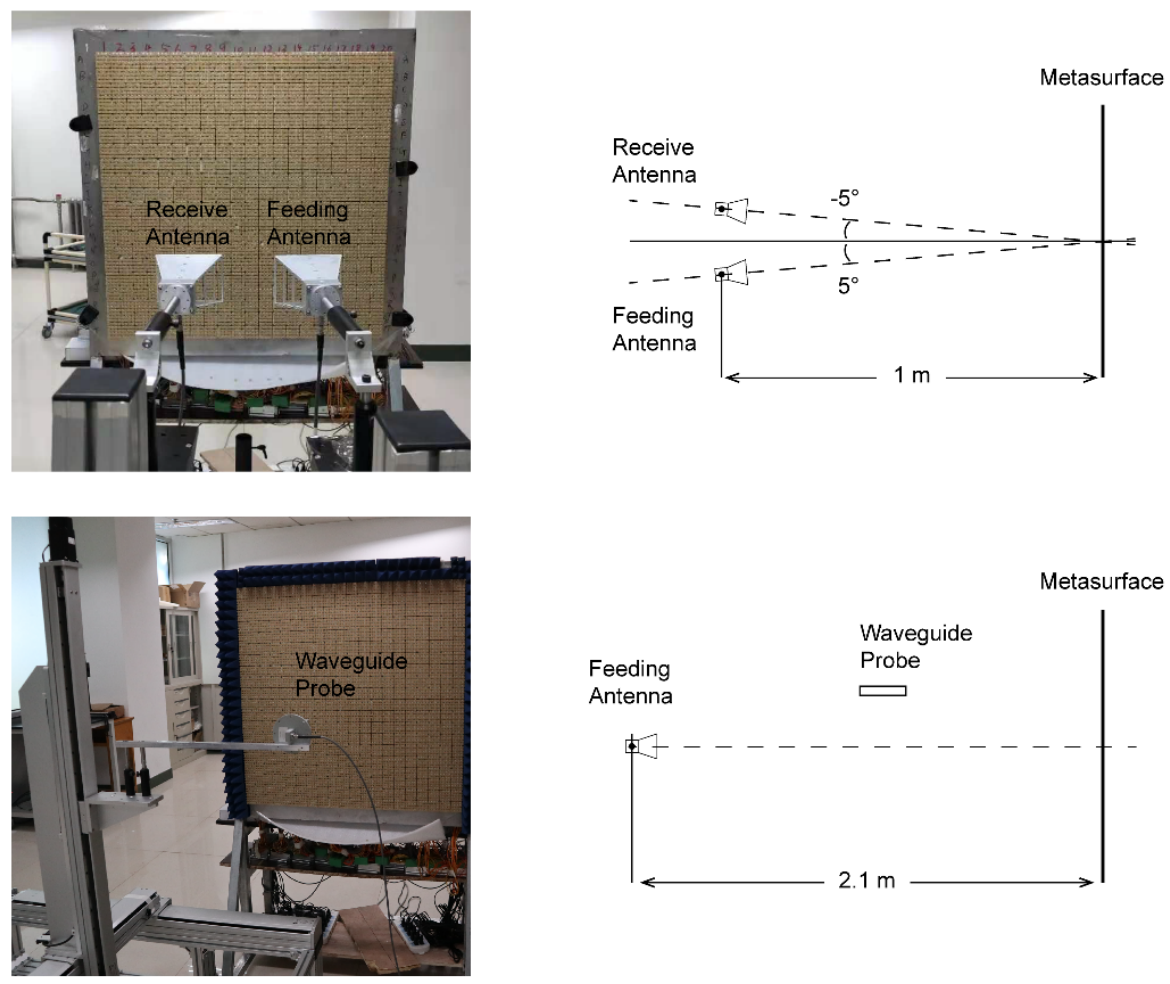

Figure S17. (a) Results shown in Fig. 2 in the main text are measured by adopting two same broadband antennas as feeding source and receiver, respectively. These two antennas are placed with $\pm 5^{\circ}$ inclination, respectively, for the approximately measure of normally reflected signals. The distance between the antennas and metasurface is about $1 \mathrm{~m}$. (b) Results shown in Figs. 3-5 in the main text are scanned by adopting a broadband antenna as feeding source and a waveguide probe as receiver, respectively. 